\section{In vivo protein-DNA interactions in a glucocorticoid response element require the presence of the hormone}

\author{
Peter B. Becker, Bernd Gloss, Wolfgang Schmid, \\ Uwe Strähle \& Günther Schütz
}

Institute of Cell and Tumor Biology, German Cancer Research Center, Im Neuenheimer Feld 280, D-6900 Heidelberg, FRG

\begin{abstract}
Transcriptional activation of gene expression by glucocorticoid hormones is mediated by the interaction of hormone-receptor complexes with specific DNA sequences called glucocorticoid responsive elements (GREs) (refs 1-3, see ref. 4 for review). Deletion of this sequence abolishes glucocorticoid induction of transcription ${ }^{4-8}$. According to a current model, activation of the cytoplasmic receptor protein by hormone binding leads to its increased affinity for and translocation to the nucleus 4 . However, recent reports that the oestradiol and progesterone receptors are localized in the nucleus in the absence of steroid ${ }^{9-11}$ led us to examine whether the free receptor interacts in vivo with its DNA binding site in the absence of hormone binding. We used the genomic footprinting technique ${ }^{12-15}$ to show that changes in in vivo protein-DNA interactions within the GREs of the tyrosine aminotransferase gene (TAT) can be detected only after hormone treatment in hepatoma cells. Such changes are not detected in fibroblast cells, in which the TAT gene is not expressed. Many of the changes in dimethylsulphate reactivity observed in the living cell are also found in vitro using cloned DNA and a partially purified glucocorticoid receptor.
\end{abstract}

The GREs of the rat TAT gene are located unusually far upstream, at -2.5 kilobases $(\mathrm{kb})$, from the transcriptional start site (Fig. 1). These elements were initially indicated by the appearance of a DNaseI-hypersensitive site following hormone treatment ${ }^{16}$ and then identified by gene transfer experiments and DNaseI footprinting using partially purified glucocorticoid receptor complex in vitro ${ }^{17}$. Sequence analysis revealed several sequence motifs that fit the established GRE consensus sequence ${ }^{1,2,4}$. In this study we focus on those elements (II and III, Fig. 1) that have been shown to be essential for glucocorticoid induction by gene transfer experiments.

The instability during purification of the glucocorticoid receptor in the absence of ligand has hindered the analysis of its potential interaction with DNA sequences by in vitro methods, but the recent development of the genomic footprinting technique $^{12,15}$ has made possible the analysis of receptor-DNA interactions in vivo in the absence of steroid. Cells of the $T A T$-expressing hepatoma cell line FTO-2B were withdrawn from glucocorticoids by incubation for at least 16 hours in serum-free medium before induction with the steroid dexamethasone for various times. Trypsinized cells suspended in medium were reacted with dimethyl sulphate (DMS) to probe for the reactivity of the $\mathrm{N} 7$ position of guanines in the major groove of the DNA helix ${ }^{15,19,20}$. Patterns of guanine $(G)$ residues were obtained using the probe fragment indicated in Fig. 1.

Figure $2 a$ shows an example of such an analysis of uninduced and induced FTO cells. The band intensity reflects the reactivity of a given guanine or a group of unresolved guanines for the chemical modification. Protection from methylation at guanines is most readily explained by protein binding whereas enhanced modification of guanines has been interpreted as a local increase in reagent concentration in a hydrophobic pocket caused by close contacts of protein to $\mathrm{DNA}^{20}$. Methylation protection and enhancement after hormone treatment were analysed quantitatively for each guanine (Fig. $2 c$ ). The data were collected from four independent experiments, each DNA sample being analysed on at least two blots. A number of changes in band intensity

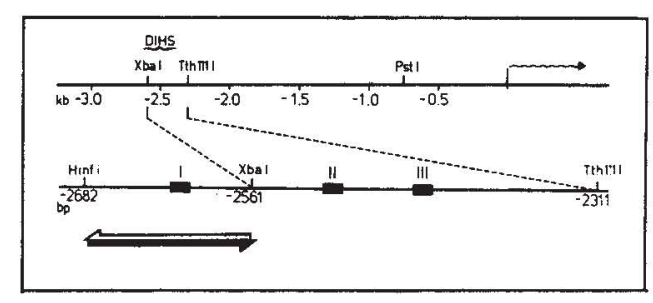

Fig. 1 The 5'-flanking region of the rat TAT gene. Upper line, $3 \mathrm{~kb}$ of the 5 -flanking region of the rat $T A T$ gene: Numbering, distance from the cap-site; wavy arrow, the start of transcription; DIHS, region of dexamethasone-induced DNaseI hypersensitivity ${ }^{16}$. The lower line, enlargement of the region around the dexamethasoneinducible DNaseI-hypersensitive site: black boxes, sequence homologies to the GRE consensus sequence labelled with roman numerals; arrows, single-stranded DNA probes used to generate genomic sequence synthesized from an M13 vector containing the HinfI-XbaI fragment.

appear to be correlated with hormone treatment. DMS protections are evident at the sites of glucocorticoid interaction as identified by DNasel footprinting ${ }^{17}$. Additional changes in reactivity map to neighbouring sequences (see Fig. 4). In a nonexpressing rat fibroblast cell-line (XC cells) no such changes could be discerned after hormone treatment (Fig. $2 a$ ), nor were they observed when FTO-2B cells were treated with the glucocorticoid antagonist RU486 (Fig. $2 b$ ).

To ascertain whether the changes in DMS reactivity observed in vivo at the TAT gene GREs are caused by the glucocorticoid receptor, we performed in vitro methylation experiments, using cloned TAT DNA fragments containing the GREs and a partially purified glucocorticoid receptor (Fig. 3). The most prominent $G$ protections map to the conserved TGTTCT motif and are identical with those shown to be contacted by the receptor in the GRE of mouse mammary tumour virus (MMTV) ${ }^{21}$.

Figure 4 summarizes the data obtained in vivo and in vitro. Comparison of in vivo $\mathrm{G}$ reactivity patterns from uninduced and induced FTO-2B cells clearly shows that protection of guanines in the binding site is seen after dexamethasone treatment of cells (Fig. 2). They are accompanied by a number of hormone-induced changes in reactivity in neighbouring sequences. The most dramatically enhanced $G$ reactivities are seen at four adjacent guanines $(-2,459$ to $-2,456)$ between the two receptor binding sites. Most of the changes in DMS reactivity following hormone treatment are also found in vitro using a partially purified glucocorticoid receptor. The changes are not found in XC cells, where the TAT gene is not transcribed. These findings strongly indicate that changes in reactivity at guanines within the GRE are indeed caused by interaction of the glucocorticoid receptor with its binding site and that its affinity in the absence of hormone binding is too low to lead to a tight interaction with a GRE at receptor concentrations found in the living cell.

Receptor binding is accompanied by changes in chromatin structure that render a region of 200 base pairs (bp) surrounding the GREs hypersensitive to DNaseI digestion ${ }^{16}$. Whether this altered structure is a prerequisite for receptor binding or rather a consequence of binding is not known. For the MMTV GREs a similar DNaseI hypersensitivity has been shown to precede or parallel hormonal stimulation of MMTV promoter function ${ }^{18}$.

It should be emphasized that these effects on guanine reactivities have been observed within a region which is unusually remote (at $-2.5 \mathrm{~kb})$ from the cap-site that is selectively rendered hypersensitive to DNase I digestion after dexamethasone treatment. Using the same DNA samples no hormone-dependent DMS effects were seen within several hundred nucleotides surrounding the TAT gene cap-site or within a region of DNase I hypersensitivity at $-1,000 \mathrm{bp}$ (data not shown). Within the first 
Fig. 2 Glucocorticoid-induced changes in DMS reactivity in vivo $a, \mathrm{G}$ ladders for both strands of a region of genomic $\mathrm{DNA}$ between 2,380 and $-2,540$ bp (upstream) of the transcriptionat start site of the TAT gene from DMS-treated FTO-2B or XC cells (+ induced with dexamethasone; -, uninduced); $N$, in vitro methylated protein-free DNÄ. Protection $(\triangle)$ and enhancement $(\Delta$ seen when comparing dexamethasone-induced with uninduced FTO-2B cells; numbers, distance from the cap-site. Only those reactivity changes that appeared highly significant in the quantitative analysis $(c)$ are indicated. *, Band originating from hybridization to lacZ sequences on a contaminating fragment; hatched bars, positions of the two major DNaseI footprints obtained in vitro with partially purified glucocorticoid receptor ${ }^{17} . b$, As in $a$, but only FTO-2B cells were used and incubation with the glucocorticoid antagonist RU486 (RU) was carried out in parallel with dexamethasone inductions. $c$, Quantitative analysis of hormonedependent changes in $\mathrm{G}$ methylation; summary of results. Nucleotide numbers (from $-2,560$ to $-2,390$ ) upstream of the TAT cap-site are given on the abscissa. Hatched bars, sites of receptor binding identified by DNaseI footprinting in vitro ${ }^{17}$; black bar conserved hexanucleotide TGTTCT. For each $\mathrm{G}$ residue the logarithm between the median of induced divided by the median of uninduced samples $(\log +\operatorname{dex} /-$ dex $)$ is plotted. $\Delta$ and $\nabla$ symbolize this value for each guanine on the upper and lower strand, respectively. Positive values indicate dexamethasonedependent enhancements, negative values represent inducible protections. The significance of the deviations obtained was checked by comparing induced and uninduced values using a KruskalWallis test ${ }^{24}$. Those values that fell below a threshold of $5 \%$ probability of error (1st order error) are indicated with vertical lines.

Methods. FTO-2B and XC cells were grown to about $80 \%$ confluency in Dulbecco's modified Eagle's medium (DMEM) contain ing $10 \%$ fetal calf-serum and $10 \mathrm{mM}$ HEPES $\mathrm{pH} 7.4$, withdrawn in serum-free DMEM for at least $16 \mathrm{~h}$ and incubated for $4 \mathrm{~h}$ with serum-free DMEM with or without $10^{6} \mathrm{M}$ dexamethasone or $10^{-6} \mathrm{M}$ RU486. Induction times were $20 \mathrm{~min}-12 \mathrm{~h}$, no significant time-dependent changes in the DMS reactivity pattern were observed (data not shown). DMS treatment of whole cells and in vitro methylation of protein-free DNA were as published ${ }^{15,19}$ After isolation of cellular DNA, $25 \mu \mathrm{g}$ of each sample was restric
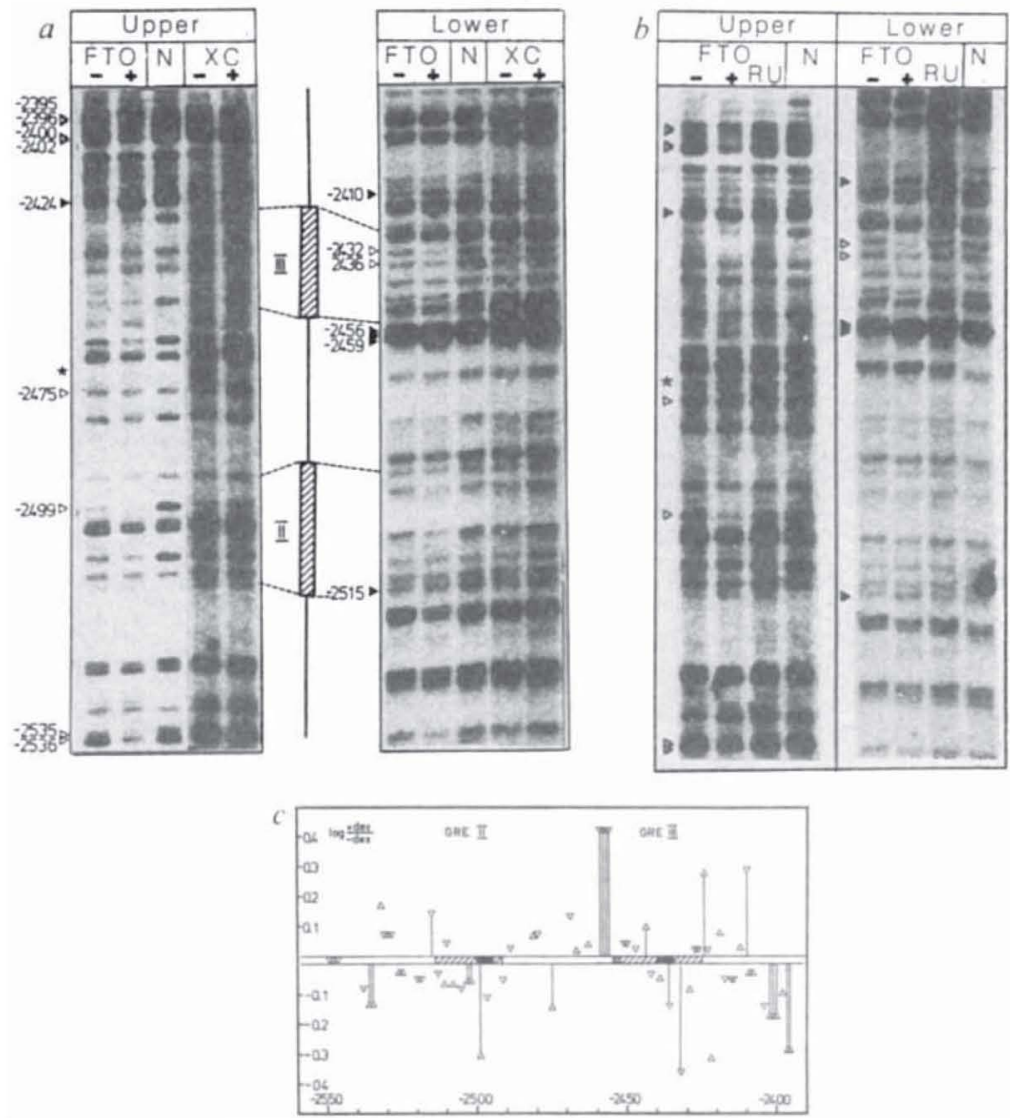

ted with Hinfl (Boehringer), reacted with piperidine and separated on a sequencing gel ${ }^{12}$. Details of DNA blotting onto Gene Screen membrane (NEN) UV crosslinking, hybridization and washing of filters were as in ref. 12. Probe synthesis from a single-stranded M13 template was performed as described ${ }^{12}$ with modifications: $100 \mathrm{mM} \mathrm{NaCls}$ was included in primer annealing and elongation reactions, $250 \mu \mathrm{Ci}$ of $\left[\alpha^{32} \mathrm{P}\right] \mathrm{dATP}(\mathrm{NHN}), 5,000 \mathrm{Ci}$ mmole ${ }^{-1}$ was used per probe synthesis. The newly-synthesized probe INNA was separated from the template strand in a $6 \%$ denaturing gel and recovered from the polyacrylamide by isotachophoresis ${ }^{22}$. Exposure times were 7-14 days with 'lightning plus' intensifying screens (DuPont). Quantitative analysis was done as Giniger et al. ${ }^{23}$; four independent DMS treatments of FTO-2B dells were performed under either dexamethasone-induced or uninduced conditions. The resulting DNAs were analysed on at least two independent blots. Genomic sequencing ladders were scanned with an Elscript 400 densitometer (Fisher Scientific) at $16 \mu m$ resolution. Peak integrals were calculated and median values determined for each guanine band.

Fig. 3 Changes in DMS reactivity following glucocorticoid receptor binding in vitro. L.eft panel, results obtained for the upper strand and right panel, for the lower strand with $(+)$ and without $(--)$ triamcinolone-saturated glucocorticoid receptor. Protected guanines are indicated by open squares, enhanced methylation at a guanine as a filled square. Guanines that are influenced in their reactivity when comparing the "-' and '+' lanes are labelled with their numbers upstream of the TAT cap-site. Hatched bars, regions protected against DNaseI-digestion by bound glucocorticoid receptor in vitro ${ }^{17}$. Methods. Purification of the glucocorticoid receptor ${ }^{25}$ included as a final step DEAE-Sepharose 6 CL column chromatography in which the receptor eluted as a single peak at $150 \mathrm{mM} \mathrm{NaCl}$. A $X b a I-T t h 111$ f fragment containing both functional GREs was isolated from a subclone and endlabelled using T4 kinase polynucleotide and $\left[\gamma^{32}\right.$ P]ATP either at the XbaI (upper strand) or at the Tth 1111 (lower strand) sites. Labelled fragment $(0.5-1.0 \mathrm{fmol})$ was incubated with $(+)$ or without (-) 90 fmoles of triamcinolone-saturated receptor in a volume of $25 \mu \mathrm{l}$ containing $100 \mathrm{mM} \mathrm{NaCl}$,

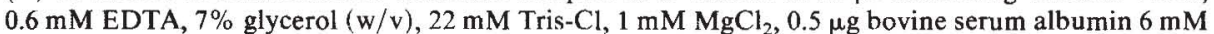
$\beta$-mercaptoethanol, $0.1 \mathrm{mM}$ dithiothreitol, $1 \mu \mathrm{M}$ triamcinolone, $15 \%$ polyethyleneglycol 6000 (Serva), $p \mathrm{H}$ 8.2. After $45 \mathrm{~min}$ at room temperature, $2 \mu \mathrm{l}$ of $10 \%$ DMS (Fluka) was added and reaction stopped after $2 \mathrm{~min}$ incubation at room temperature by adding $75 \mu 1$ buffer containing $400 \mathrm{mM}$ sodium acetate, $140 \mathrm{mM} \beta$-mercaptoethanol and $270 \mu \mathrm{g} \mathrm{ml}^{-1}$ transfer RNA. DNA was extracted with phenol/chloroform $(1: 1)$, precipitated and subjected to piperidine cleavage according to Maxam and Gilbert ${ }^{19}$, and finally dissolved in formamide containing loading buffer and analysed on a $6 \%$ sequencing gel.

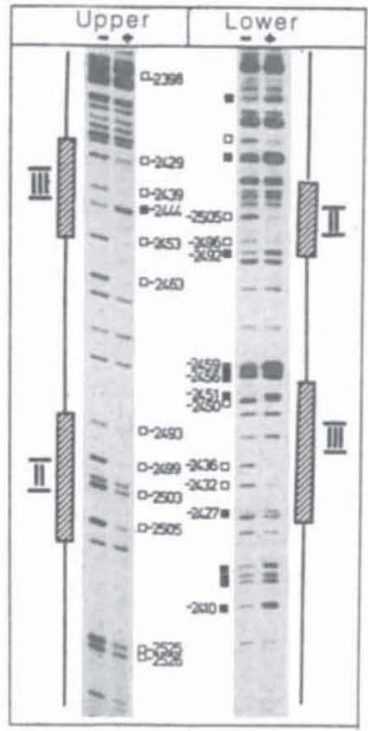

200 bp upstream of the transcriptional start site several protections and enhancements can be identified that are strictly correlated with the active state of the gene in FTO-2B cells but are not seen in XC cells. However, none of them are influenced by hormone treatment (P.B., unpublished data).

Comparison of the methylation patterns of the two analysed GREs in vivo reveals qualitative and quantitative differences.
Whereas the protection of the central guanine $(-2,499)$ in element II is clearly evident, it is less prominent in element III $(-2,436)$. A surprising finding is a very strong protection of a TGT $(-2,432)$ upstream of the conserved hexanucleotide TGTTCT of GRE III. Why the two binding sites behave so differently is not known. Recent results suggest that this difference in DMS reactivities might reflect differences in the 


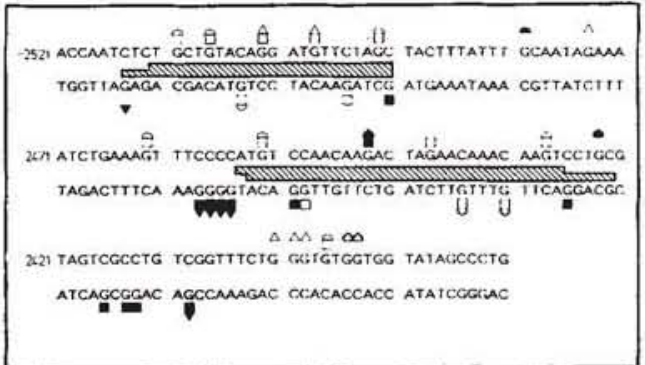

Flg. 4 Summary of results obtained by DMS reactivity experiments in vivo and in vitro. Changes in DMS reactivity at guanines in the DNA sequence between nucleotides $-2,521$ and $-2,382$, upstream of the TAT cap-site, are shown. Hatched bars, sites of glacocorticoid receptor interaction as identified by in vitro footprinting experiments ${ }^{17}$. Filled symbols, G residues showing enhanced methylation; open symbols, guanines showing protections from methylation. Squares, in vitro data; triangles, dexamethasone-dependent changes in vivo; half-circles, changes in reactivity found in vivo comparing the pattern obtained from uninduced cells with that from protein-free DNA. For the in vivo data only those guanines are indicated that by quantitative analysis have been shown to be significantly influenced in their reactivity (Fig. 2c).

inducing capacity of the two GREs (M. Jantzen and U.S., unpublished data).

In addition to effects in the receptor binding site, a number of changes in reactivity are observed in neighbouring sequences after dexamethasone treatment. Most prominent among these are the enhancements of guanines at $-2,515,-2,459$ to $-2,456$, and at $-2,424$ and protections of guanines at $-2,475,-2,402$ to $-2,400,-2,396$, and $-2,395$, in vivo and to some extent in vitro. Whether these changes are due to alterations in DNA structure as a consequence of glucocorticoid receptor binding or interaction with other factors not yet defined remains unclear.

These changes in DMS reactivity are clearly correlated with hormone induction and other changes are evident when the pattern of DMS reactivity of uninduced FTO-2B cell chromatin is compared with that of protein-free FTO-2B DNA (quantitative analysis not shown). Analysis of the reactivity of single guanines in naked genomic DNA at various sites and in plasmid DNA revealed that guanines flanked by thymidines or followed by a thymidine are often unusually sensitive to methylation. This enhanced reactivity is not observed in vivo, which might be explained by the difference in reaction conditions inside living cells compared with protein-free DNA in vitro. Because of these differences in methylation conditions naked genomic DNA cannot serve as a suitable standard for comparisons of DMS reactivity obtained in vivo. The methylation pattern in uninduced FTO-cells is identical to that obtained from induced or uninduced XC-cells. In contrast to observations at the cap-site, there is no evidence for an interaction of protein factors with the receptor binding site in the absence of hormone that discriminates $T A T$ in FTO-cells from the gene in nonexpressing fibroblast cells.

We have applied genomic sequencing methodology to obtain information on the in vivo interaction of the glucocorticoid receptor with its recognition sequence in the $T A T$ gene. Results support the concept that glucocorticoid increases the affinity of the receptor for its target sequence. These experiments do not exclude the possibility that the unliganded receptor can interact with a GRE, albeit with lower affinity and selectivity.

We thank Harry Nick for introducing us to the genomic sequencing methodology; Professor E. Weber for help with quantitative analysis, Alfred Nordheim, Richard Miksicek, Francis Stewart and Michael Boshart for critical reading of the manuscript and M. Cole for secretarial assistance. This work was supported by the Deutsche Forschungsgemeinshaft (Schu 51/4-2).
Received 23 June; accepted 28 October 1986.

1. Payvar, F. P. et at Cell 35, 38!-392 (198.3).

2. Scheidereit, C., Geisse, S. Westphal, H. M \& Beato, M. Nature 304, 749-752 (1983)

3. Van der Ahe, D. et al. Nature 313, 706709 (1985)

4. Yamamoto. K. R. A. Ret, Genet, 19, 209-252 (1985)

5. Hynes, N. et. al. Proc. natn. Acad. Sci. U.S.A. 80, 3637, 3641 (1983

6. Renkawitz, R., Schütz, G., von der Ahe, D. \& Beato, M. Cell 37, 503-510 (1984)

7. Karin, M. et at Nature 308, 513.519 (1984)

8. Miksicek, R. et al. Cell 46, $283,290(1986)$

9. King, W. J. \& Greene, G. L. Nature 307, 745-747 (1984

. Welshons, W. K., Liebermann, M. E. \& Gorski, J. Nature 397, 747749 (1984).

1. Perrot-Applanat, M., Logeat, 1., Groyer-Picard, M. T. \& Milgrom, F. Lndocrinology 116, 1473-1484 (1985)

12. Church, G. M. \& Gilbert, W Proc natn, Acad Sci U.S.A. 81, 1991-1995 (1984)

13. Church, G. M., Ephrussi, A., Gilbert, W. \& Tonegawa, S. Nature 313, 798-801 (1985)

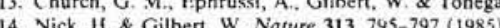

15. Eichrussi, A. Church, G. M. Tonegawa, S. \& Gilbert, W. Science 227, 134-140 (1985)

16. Becker, P. B. Renkawitz, R. \& Schutz, G. EMBO J 3, 2015-2020 (1984).

17. Janteen, M. et al. (in preparation).

18. Zaret, K. S. \& Yamamoto, K. R. (ell 38, 29-38 (1984)

9. Maxam, A. M. \& Gilhert, W. Meth. Fnzym. 65, 499 (1978)

20. Ogata, R. T. \& Gilbert, W. Proc. natm. Acad, Sct. U.S. A. 75, 58515854 (1978)

1. Scheidereit, C. \& Beato, M. Prac nain. Acad Sci. L.S.A. 81, 3029.3033 (1984)

22. Öfverstedt, L. (i. et al Biochim, biophys. Acta 782, 120-126 (1984)

23. (Gininger, E., Varnum, S. M. \& Ptashne, M. Cell 40, 767-774 (1985)

24. Kruskal, W. H. Ann, math Statist. 23, 525-540 (1952).

25. Singh, V. B. \& Moudgil, V K. J. hiol. Chem 260, 3684-3690 (1985) 